\title{
ON THE DIFFERENTIAL PROPERTIES OF CONTINUOUS FUNCTIONS
}

\section{ПРО ДИФЕРЕНЩАЛЬНІ ВЛАСТИВОСТІ НЕПЕРЕРВНИХ ФУНКЩЙ}

We introduce and investigate some new differential properties of continuous functions by means of the geometrical properties of their derivatives.

Введено та досліджено деякі нові диферешціальні властивості пеперервпих фупкцій за допомогого геометричних властивостей їх похідних.

1. Preliminaries. It is usual to say that a "typical" element of some Baire space has a certain property if the set of elements that do not possess this property is of the first Baire category [1]. Then we also say that "most" elements have the given property.

It is known, [2], that typical real continuous functions of one real variable have no finite unilateral derivative at any point [1], have no infinite derivative at any point, [3], but have an infinite unilateral derivative at a set not of the first Baire category [4].

Let $I=[0,1]$. It is known [2] that the space $C$ of all real continuous functions of one real variable $f: I \rightarrow R$, endowed with the usual distance $\sup _{x \in I}|f(x)-g(x)|$ between $f, g \in C$ is a Baire space. The subspace $M \subset C$ of all increasing functions in $C$ is also a Baire space.

For $f \in C, x \in I$, the set $M_{x}(f)$, right set $M_{x}^{\prime}(f)$, and left set $M_{x}^{\prime \prime}(f)$ of derived numbers are defined, respectively, as follows:

$$
\begin{aligned}
& M_{x}(f)=\bigcap_{\varepsilon>0} \overline{M_{\varepsilon}(x)}, \\
& M_{x}^{\prime}(f)=\bigcap_{\varepsilon>0} \overline{M_{\varepsilon}^{\prime}(x)}, \\
& M_{x}^{\prime \prime}(f)=\bigcap_{\varepsilon>0} \overline{M_{\varepsilon}^{\prime \prime}(x)}
\end{aligned}
$$

where the overbar denotes the closure operators, $M_{\varepsilon}^{\prime}(x)\left[M_{\varepsilon}^{\prime \prime}(x)\right]$ is the set of values of the increment ratio $\frac{\Delta f}{\Delta x}$ for all $\Delta x, \Delta x \in(0, \varepsilon)[\Delta x \in(-\varepsilon, 0)]$ and $M_{\varepsilon}(x)=$ $=M_{\varepsilon}^{\prime}(x) \cup M_{\varepsilon}^{\prime \prime}(x)$.

For any function $f \in C$, let $f_{i}^{\prime}$ be the lower, $f_{s}^{\prime}$ the upper, $f_{i}^{-}$the left lower, $f_{s}^{-}$the left upper, $f_{i}^{+}$the right lower, and $f_{s}^{+}$the right upper Dini derivatives of $f$. Thus, we have $f_{i}^{\prime}, f_{s}^{\prime} \in M_{x}(f), f_{i}^{-}, f_{s}^{-} \in M_{x}^{\prime \prime}(f), f_{i}^{+}, f_{s}^{+} \in M_{x}^{\prime}(f)$.

We recall the following known results.

Theorem A [2]. For a typical function $f \in C$, at each point $x \in I$, we have $-\infty \in M_{x}(f)$ and $+\infty \in M_{x}(f)$.

Theorem B [1]. For a typical function $f \in C$, at each point $x \in(0,1]$, we have $-\infty \in M_{x}^{\prime \prime}(f)$ or $+\infty \in M_{x}^{\prime \prime}(f)$ and, at each point $x \in[0,1)$, we have $-\infty \in \in M_{x}^{\prime}(f)$ or $+\infty \in M_{x}^{\prime}(f)$.

Theorem C [4]. For a typical function $f \in M$, at each point $x \in(0,1]$, we have $0 \in M_{x}^{\prime \prime}(f)$ or $+\infty \in M_{x}^{\prime \prime}(f)$ and, at each point $x \in[0,1)$, we have $0 \in$ $\in M_{x}^{\prime}(f)$ or $+\infty \in M_{x}^{\prime}(f)$.

Theorem $\mathrm{C}$ has the following immediate consequences. 
Corollary A [4]. For a typical function $f \in M$, we have $M_{x}(f)=\{0\}$ a. e.

Corollary B. For a typical function $f \in M$, we have $M_{x}(f)=\{\infty\}$ at densely, uncountably many points $x \in I$.

Proof. Since $f$ is strictly increasing, there exists $f^{-1}$, which being monotone, is differentiable a. e. But $f$ has in no point of $I$ a finite derivative different from zero, by theorem C. Hence, $\left(f^{-1}\right)^{\prime}=0$ a. e., whence $M_{x}(f)=\{\infty\}$ at densely uncountably many points $x \in I$.

A function $f \in M$ is called nonangular if

$$
f_{i}^{-1} \leq f_{s}^{+} \text {and } f_{i}^{+} \leq f_{s}^{-} \text {. }
$$

The following result is known [1]:

Theorem D. A typical function in $C$ is nonangular.

It is not hard to show that the same is true for monotone continuous functions and that the following theorem holds:

Theorem 1. A typical function in $M$ is nonangular.

There is an analogy between the properties of typical continuous and typical monotone increasing functions. This is evident by comparing Banach's theorem B with theorem $\mathrm{C}$, and theorem $\mathrm{D}$ with Theorem 1 .

Now we prove the following theorem.

Theorem 2. For a typical function $f \in C$, we have

$$
-\infty \in M_{x}^{\prime}(f) \cap M_{x}^{\prime \prime}(f), \quad+\infty \in M_{x}^{\prime}(f) \cap M_{x}^{\prime \prime}(f)
$$

at most points $x \in I$.

Proof. In [2] it was proved that, for any function $g \in C$, we have $g_{i}^{-}(x)=g_{i}^{+}(x)$ and $g_{s}^{-}(x)=g_{s}^{+}(x)$ at most points $x \in I$. Now by theorem $\mathrm{A}$ above we get the required result.

2. Main results.

Theorem 3. For a typical function $f \in M$, we have $0 \in M_{x}^{\prime}(f) \cap M_{x}^{\prime \prime}(f)$ and $\infty \in M_{x}^{\prime}(f) \cap M_{x}^{\prime \prime}(f)$ at most points $x \in I$.

Proof. Let $f \in M$ and

$$
A=\left\{x-\in(0,1): f_{i}^{+}(x)=0 \text { and } f_{s}^{+}(x)=\infty\right\} .
$$

For $x \in(0,1)$, we set

$$
\begin{aligned}
& f_{+}(x)=\sup _{0<h<\varepsilon} \frac{f(x+h)-f(x)}{h} \in M_{x}^{\prime}(f), \\
& f_{-}(x)=\inf _{0<h<\varepsilon} \frac{f(x+h)-f(x)}{h} \in M_{x}^{\prime}(f) .
\end{aligned}
$$

We see that

$$
A=\left\{x \in(0,1): f_{-}(x)=0 \text { and } f_{+}(x)=\infty\right\} .
$$

Let $F$ be the family of all functions $f \in M$ such that $A$ is not residual. Consider $f \in$ $\in F$ and write

$$
A_{n}=\left\{x \in(0,1): f_{-}(x)<1 / n \text { and } f_{+}(x)>n\right\} \text {. }
$$

Obviously, $x_{m} \rightarrow x$ and $f_{+}\left(x_{m}\right) \leq n$ imply that $f_{+}(x) \leq n$. Also, from $f_{-}\left(x_{m}\right) \geq 1 / n$, we get $f_{-}\left(x_{m}\right) \geq 1 / n$.

Thus, for $x_{m} \rightarrow x, f_{+}\left(x_{m}\right) \leq n$ or $f_{+}\left(x_{m}\right) \geq 1 / n(m \in \mathbb{N})$, it results that $f_{+}(x) \leq n$ or $f_{-}(x) \leq 1 / n$. Hence, $I / A_{n}$ is closed. 
Since $A=\bigcap_{n=1}^{\infty} A_{n}$ is not residual, the set $I / A=\bigcup_{n=1}^{\infty}\left(I / A_{n}\right)$ is not of the first category. It follows that the set $I / A_{n}$ is not nowhere dense for some $n \in \mathbb{N}$, i. e., $I / A_{n}$ includes an interval. Let $F_{m, n}$ be the set of all functions $f \in F$, such that $I / A_{n}$ includes, for some index $n$, an interval of length $1 / m(m, n \in \mathbb{N})$. It is easy to show that each of the sets $F_{m, n}$ is closed.

To prove that $M / F_{m, n}$ is dense, one can approximate any function $f \in M$ by piece wise linear, strictly increasing functions $g: I \rightarrow R$. By replacing appropriately linear restrictions $\left.g\right|_{[a, b]}$ of $g$ (with $b-a<1 / m$ ) by increasing functions $h:[a$, $b] \rightarrow R$ defined so that $h(a)=g(a), h(b)=g(b), h_{i}^{+}(a)=0, h_{s}^{+}(a)=\infty$, with $\max |h-g|([a, b])$ small enough, we find functions approximating $f$ in $M / F_{m, n}$. Now it is shown that each $F_{m, n}$ is nowhere dense, i. e., $F=\bigcup_{m, n} F_{m, n}$ of the first category.

Hence, for a typical $f \in M$, the set $A$ is residual; analogously, the set

$$
B=\left\{x \in(0,1): f_{i}^{-}(x)=0 \text { and } f_{s}^{-}(x)=\infty\right\}
$$

is also residual, which shows that $A \cap B$ is residual.

Hence, contrary to the measure - theoretical point of view (Lebesque's theorem), we have the following result:

Corollary. A typical functions $f \in M$ is not differentiable at most points of $I$.

For the last two theorems we need the following simple lemma:

Lemma. Let $-\infty \leq \alpha<\beta \leq \infty$. If $f \in C$ and $f_{i}^{+}(x)=\alpha$ and $f_{s}^{+}(x)=\beta$, $\alpha, \beta \in M_{x}^{\prime}(f)$ at a dense set of points $x$, then for each $k \in(\alpha, \beta)$, there are two dense sets $A_{k}$ and $B_{k}$ such that $f_{s}^{-}(x) \leq k \leq f_{i}^{+}(x)$ for $x \in A_{k}$ and $f_{s}^{+}(x) \leq$ $\leq k \leq f_{i}^{-}(x)$ for $x \in B_{k}$

Proof. We prove the existence of $B_{k}$. Let $z \in I$. There is a point $x \in[0,1)$ as close to $z$ as we want such that

$$
f_{i}^{+}(x)=\alpha \text { and } f_{s}^{+}(x)=\beta .
$$

Let $\alpha<a<k<b<\beta$. We can choose $x_{1}, x_{2}$ arbitrarily close to $x$ such that $\frac{f\left(x_{1}\right)-f(x)}{x_{1}-x} \leq a, \quad \frac{f\left(x_{2}\right)-f(x)}{x_{2}-x} \geq b$. Then exists $x_{3} \in\left(x_{1}, x_{2}\right)$ such that $\frac{f\left(x_{3}\right)-f(x)}{x_{3}-x}=k$. Let $y$ be an absolute maximum of $\left.g\right|_{\left[z, x_{3}\right]}$, where $g(t)=f(t)-$ -kt. Obviously, $g_{i}^{-}(y) \geq 0$ and $g_{s}^{+}(y) \leq 0$, whence $f_{i}^{-}(y) \geq k$ and $f_{s}^{+}(y) \leq k$. Analogously, the existence of $A_{k}$ can be verified.

Theorem 4. For a typical function $f \in C$ and any $k \in R$, there exist two dense sets $A_{k}$ and $B_{k}$ such that

$$
\begin{aligned}
& f_{i}^{-}(x)=-\infty, \quad f_{s}^{-}(x)=f_{i}^{+}(x)=k, \quad f_{s}^{+}(x)=\infty \text { for } x \in A_{k}, \\
& f_{i}^{+}(x)=-\infty ; \quad f_{s}^{+}(x)=f_{i}^{-}(x)=k, \quad f_{s}^{-}(x)=\infty \text { for } x \in B_{k} \text {. }
\end{aligned}
$$

Proof. By Theorem 2 and the lemma. with $a=-\infty$ and $b=\infty$, for each $k \in R$, there exist dense sets $A_{k}, B_{k}$ such that

$$
f_{s}^{-}(x) \leq k \leq f_{i}^{+}(x) \text { for } x \in A_{k} \text { and } f_{s^{-}}^{-}(x) \leq k \leq f_{i}^{+}(x) \text { for } x \in B_{k} \text {. }
$$


Now, in view of Theorems $\mathrm{B}$ and $\mathrm{D}$, it is obvious that the inequalities of the theorem hold.

Theorem 5. For a typical function $f \in M$ and any $k>0$, there exist two dense sets $A_{k}$ and $B_{k}$ such that.

$$
\begin{aligned}
& f_{i}^{-}(x)=0, \quad f_{s}^{-}(x)=f_{i}^{+}(x)=k, \quad f_{s}^{+}(x)=\infty \text { for } x \in A_{k}, \\
& f_{i}^{+}(x)=0, \quad f_{s}^{+}(x)=f_{i}^{-}(x)=k, \quad f_{s}^{-}(x)=\infty \text { for } x \in B_{k} .
\end{aligned}
$$

Proof. The argument is analogous to the preceding one and uses Theorem 3. the lemma with $\alpha=0$ and $\beta=\infty$, Theorem $C$, and Theorem 1 .

Conclusion. We note that the Dini derivatives of a typical monotone continuous function are exactly determined at almost all points, at most points, and some other dense sets of points.

1. Bruckner A. M. Differentiation of real functions. - Providence: Amer. Math. Soc., 1978. - 196 p. (CRM Monograph Ser.; Vol. 5).

2. Neugebauer C. A theorem on derivatives // Acta Sci. Math. (Szeged). - 1962. - 23. - P. $79-81$.

3. Sacks $S$. On the functions of Bezicovitch in the space of continuous functions // Fund. math. 1932. - 19. - P. $211-219$.

4. Zamfirescu T. Most monotone functions are singular // Amer. Math. Mon. - 1981. - 88. - P. 47 -49 . 